\title{
OSCILLATIONS OF A PENDULUM UNDER PARAMETRIC EXCITATION*
}

\author{
BY \\ RAIMOND A. STRUBLE \\ North Carolina State College, Raleigh, N. C.
}

1. Introduction. The motion of a simple pendulum which is excited parametrically by vertical oscillations of its support is governed by the nonlinear equation (see Fig. 1.)

$$
\theta^{*}+\left(\omega_{0}^{2}+\frac{\xi^{*}}{L}\right) \sin \theta=0,
$$

where dots indicate time differentiations, $\omega_{0}=(g / L)^{1 / 2}$ is the natural frequency of small (free) oscillations of the pendulum, $\xi$ is the displacement of the pendulum support, $L$ is the length of the pendulum and $\theta$ is the angular displacement of the pendulum from the vertical. The special case $\xi=\xi_{0} \cos \omega t$ of simple harmonic excitation was examined for periodic (subharmonic) solutions by Skalak and Yarymovych [1]. Inasmuch as their analysis was limited to the study of the periodic solutions it would, perhaps, be appropriate to present an analysis of (1) which encompasses other solutions. For the sake of brevity we will be concerned only with the primary resonant solutions when the exciting frequency $\omega$ is nearly twice the natural frequency $\omega_{0}$. Our methods, however, apply quite generally (see [2], [3], [4]).

If we introduce the dimensionless time $\tau=\omega_{0} t$ into (1) and put

$$
\epsilon=\xi_{0} / L, \quad \theta=\epsilon^{1 / 2} X, \quad \eta=\omega / \omega_{0},
$$

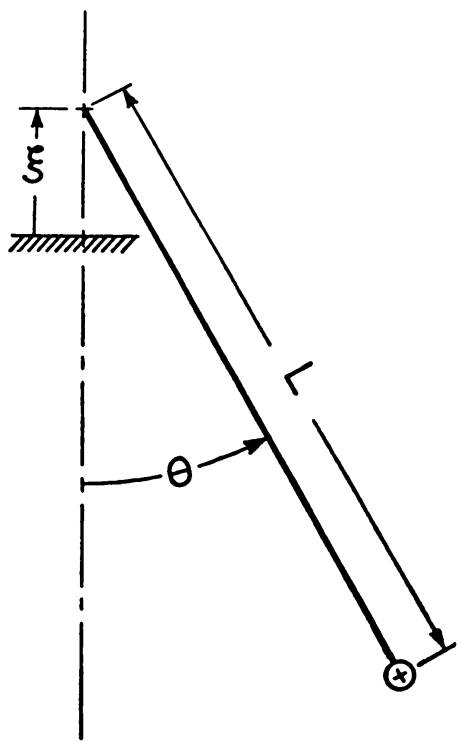

Frg. 1. Simple pendulum with oscillating support.

${ }^{*}$ Received Oct. 15, 1961; revised manuscript received Oct. 1, 1962. Work sponsored by the U. S. Army Research Office (Durham). 
we obtain the equation

$$
X^{\prime \prime}+\left(1-\eta^{2} \epsilon \cos \eta \tau\right)\left(X-\frac{\epsilon X^{3}}{6}+\frac{\epsilon^{2} X^{5}}{120}-\cdots\right)=0,
$$

where primes indicate $\tau$ differentiations. Treating $\epsilon$ as a small quantity, it is possible to develop a general perturbational solution of (2) in the form of an asymptotic* series

$$
X=a \cos (\tau-\Phi)+\epsilon X_{1}+\epsilon^{2} X_{2}+\cdots+\epsilon^{N} X_{N}
$$

in the perturbation parameter $\epsilon$. The asymptotic expansion (3) is determined by the familiar process of successive approximations, with certain innovations. For $\epsilon=0$, (2) possesses the elementary general solution $X=a \cos (\tau-\Phi)$, where the amplitude $a$ and phase $\Phi$ are arbitrary constants. For $\epsilon \neq 0$, one permits slow variations in each of $a$ and $\Phi$ and proceeds to determine these variations successively to increasing orders in potwers of $\epsilon$ together with the additive corrections $X_{1}, X_{2}, \cdots, X_{N}$. The two constants of integration for the general solution are conveniently assigned to the variable parameters $a$ and $\Phi$ and each of the additive corrections $X_{1}, X_{2}, \cdots, X_{N}$ is then expressed uniquely in terms of $a, \Phi$ and the independent variable $\tau$. We illustrate the procedure in the following sections. The leading term $a \cos (\tau-\Phi)$ of the expansion (3) is referred to as the principal term of the solution.

2. First order equations. Substituting the expansion (3) in (2) results in the equation

$$
\begin{aligned}
& {\left[2 a \Phi^{\prime}+a^{\prime \prime}-a\left(\Phi^{\prime}\right)^{2}\right] \cos (\tau-\Phi)-\left[2 a^{\prime}-2 a^{\prime} \Phi^{\prime}-a \Phi^{\prime \prime}\right] \sin (\tau-\Phi)+\epsilon\left(X_{1}^{\prime \prime}+X_{1}\right)} \\
& =\frac{\epsilon a^{3}}{8} \cos (\tau-\Phi)+\frac{\epsilon a^{3}}{24} \cos 3(\tau-\Phi)+\frac{\epsilon \eta^{2} a}{2} \cos [(1+\eta) \tau-\Phi] \\
& \quad+\frac{\epsilon \eta^{2} a}{2} \cos [(\eta-2) \tau+2 \Phi) \cos (\tau-\Phi)-\frac{\epsilon \eta^{2} a}{2} \sin [(\eta-2) \tau+2 \Phi] \sin (\tau-\Phi) \\
& +0\left(\epsilon^{2}\right),
\end{aligned}
$$

where only terms through the first power in $\epsilon$ are exhibited. In (4) we have used the elementary identity

$$
\begin{aligned}
\cos [(1-\eta) \tau-\Phi]=\cos [(\eta-2) \tau+2 \Phi] & \cos (\tau-\Phi) \\
& -\sin [(\eta-2) \tau+2 \Phi] \sin (\tau-\Phi)
\end{aligned}
$$

since we wish to examine the resonance phenomena associated with values of $\eta$ near 2 . Thus the factors $\cos (\tau-\Phi)$ and $\sin (\tau-\Phi)$ in (5) depict primary oscillations while their coefficients exhibit only slow variations.

An examination of the explicit terms in (4) suggests the following distribution

$$
\begin{aligned}
& 2 a \Phi^{\prime}+a^{\prime \prime}-a\left(\Phi^{\prime}\right)^{2}=\frac{\epsilon a^{3}}{8}+\frac{\epsilon \eta^{2} a}{2} \cos [(\eta-2) \tau+2 \Phi] \\
& 2 a^{\prime}-2 a^{\prime} \Phi^{\prime}-a \Phi^{\prime \prime}=\frac{\epsilon \eta^{2} a}{2} \sin [(\eta-2) \tau+2 \Phi]
\end{aligned}
$$

and

$$
X_{1}^{\prime \prime}+X_{1}=\frac{a^{3}}{24} \cos 3(\tau-\Phi)+\frac{\eta^{2} a}{2} \cos [(1+\eta) \tau-\Phi] .
$$

${ }^{*}$ We employ the term asymptotic as in [5]. 
Equations such as the first of these are called variational equations while an equation such as the last is called a perturbational equation. At each step of the iteration process the variational equations are associated with the fundamental harmonic terms $\cos (\tau-\Phi)$ and $\sin (\tau-\Phi)$ and the perturbational equation with the remaining nonresonant terms. Should a term appear which is "nearly" resonant, such as $\cos [(1-\eta) \tau-\Phi]$ above for $\eta$ near 2 , it is to be associated with the fundamental harmonic terms. In this way all potentially resonant or nearly resonant terms which would lead to either secular terms or small divisors in the additive perturbations $X_{1}, X_{2}, \cdots, X_{N}$ are treated in the variational equations. The additive perturbations depict "higher" harmonics of the motion and are referred to as short-period perturbations, a designation borrowed from celestial mechanics. The variables $a$ and $\Phi$ exhibit only long-period perturbations. These are usually referred to as beats.

It is readily verified that any solution of the reduced system

$$
\Phi^{\prime}=\frac{\epsilon a^{2}}{16}+\frac{\epsilon \eta^{2}}{4} \cos [(\eta-2) \tau+2 \Phi], \quad a^{\prime}=\frac{\epsilon \eta^{2} a}{4} \sin [(\eta-2) \tau+2 \Phi]
$$

satisfies (6) to first order in $\epsilon$ provided the resonance "detuning" $|\eta-2|$ is treated as a small quantity of first order in $\epsilon$. This defines the primary resonance region and (8) is then conveniently re-expressed in the autonomous form

$$
\frac{1}{\epsilon} \gamma^{\prime}=-m+\frac{a^{2}}{8}+\frac{\eta^{2}}{2} \cos \gamma, \quad \frac{1}{\epsilon} a^{\prime}=\frac{\eta^{2} a}{4} \sin \gamma,
$$

with $\gamma=(\eta-2) \tau+2 \Phi$ and $m=(2-\eta) / \epsilon$. It is clear that the variations in $a$ and $\gamma$ are slow, inasmuch as only the slow time $\epsilon \tau$ enters into (9). The long-period perturbations exhibited by $a$ and $\gamma$ (and hence $\Phi$ ) are described to first order in $\epsilon$ by (9). The corresponding short-period (additive) perturbation $X_{1}$ is described by (7). The latter, of course, need only be accurate to zero order in $\epsilon$.

3. First order solution. Stationary oscillations (3) correspond to singular (point) solutions of the variational system (9). These occur for $\sin \gamma=0$ and

$$
-m+\frac{a^{2}}{8} \pm \frac{\eta^{2}}{2}=0
$$

or, with $\eta=2-\epsilon m$, for

$$
\frac{a^{2}}{8}=m \mp \frac{1}{2}(2-\epsilon m)^{2} .
$$

This is the bifurcation equation for the quasi-harmonic system (2) and to each real positive number $a$ given by (10) there corresponds a periodic solution of (2) of amplitude approximately $a$. The frequency (in dimensionless time) of the principal term $a$ $\cos (\tau-\Phi)$ becomes $1-d \Phi / d \tau=1-(2-\eta) / 2=\eta / 2$, since $\gamma=(\eta-2) \tau+2 \Phi$ is constant. In fact, the principal term may be written in the alternate forms

$$
a \cos (\tau-\Phi)=a \cos \frac{1}{2}(\eta \tau+\gamma)=a \cos \frac{1}{2}(\omega t+\gamma) .
$$

Thus, the frequency of a periodic solution, corresponding to the bifurcation equation (10), is a $\frac{1}{2}$ subharmonic of the excitation frequency.

Nonstationary oscillations (3) correspond to nontrivial solutions of the variational 
system (9). These are readily obtained after eliminating $\tau$ in (9). Expressed in the differential form

$$
\frac{\eta^{2} a^{2}}{4} \sin \gamma d \gamma-\left(-m a+\frac{a^{3}}{8}+\frac{\eta^{2} a}{2} \cos \gamma\right) d a=0,
$$

the general first integral

$$
-m a^{2}+\frac{a^{4}}{16}+\frac{\eta^{2} a^{2}}{2} \cos \gamma=c,
$$

with $c$ an integration constant, is obtained immediately. The phase-plane curves defined by (11) are illustrated in Figures 2-6. In these plots, $\alpha=a \cos \gamma$ and $\beta=a \sin \gamma$, so that $a$ and $\gamma$ are the natural polar coordinates of the $\alpha \beta$-plane. For $c$ large, the phaseplane trajectories are very nearly the circles $a^{4} / 16=o$. As $c$ decreases, a variety of patterns are possible.

If $m<-\eta^{2} / 2=-(2-\epsilon m)^{2} / 2$, i.e. $m<\left[1-2 \epsilon+(1-4 \epsilon)^{1 / 2}\right] / \epsilon^{2} \approx-2-4 \epsilon$, then the trajectories form a family of concentric ovals closing down upon the origin as $c \rightarrow 0$. (See Fig. 2). The origin itself is therefore a center and represents a stable position of equilibrium of the pendulum. In this case, (10) yields no real values for $a$ and so there are no bifurcation amplitudes.

If $|m|<\eta^{2} / 2$, i.e. $\left[1-2 \epsilon+(1-4 \epsilon)^{1 / 2}\right] / \epsilon^{2}<m<\left[1+2 \epsilon-(1+4 \epsilon)^{1 / 2}\right] / \epsilon^{2}$, then (10) yields exactly one real positive number $a$. The corresponding bifurcation amplitude is represented by a center on the $\alpha$-axis at a distance $a$ from the origin. (See Figs. 3, 4, and 5). In addition to the larger closed trajectories, which tend to the circles $a^{4} / 16=c$ as $c \rightarrow \infty$, and the smaller closed trajectories, which tend to the center, in each instance there is an extraordinary trajectory which originates and terminates at the origin. The origin becomes an unusual singular point whose index is zero. Though it continues to represent a position of equilibrium of the pendulum, it is no longer stable.

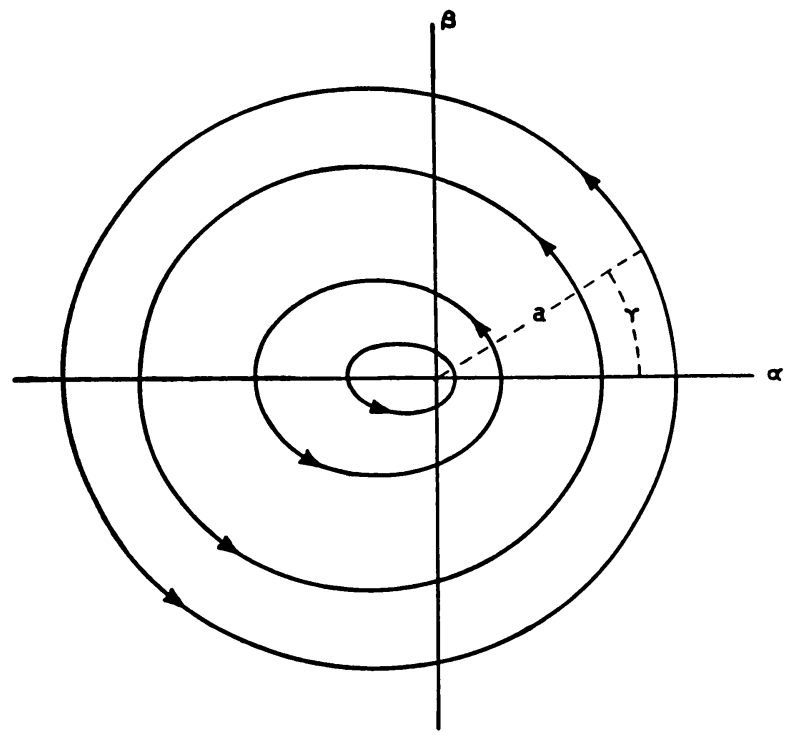

FIg. 2. Trajectories for $m<-\eta^{2} / 2$. 


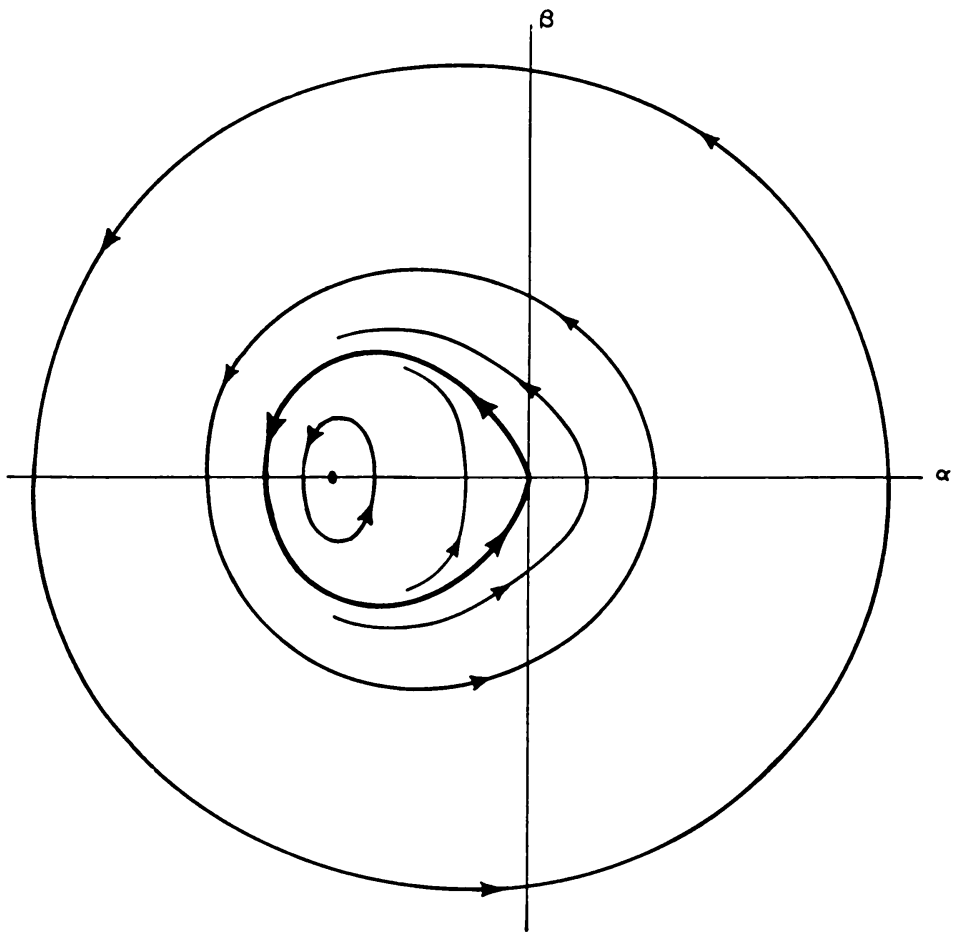

FIG. 3. Trajectories for $-\eta^{2} / 2<m<0$.

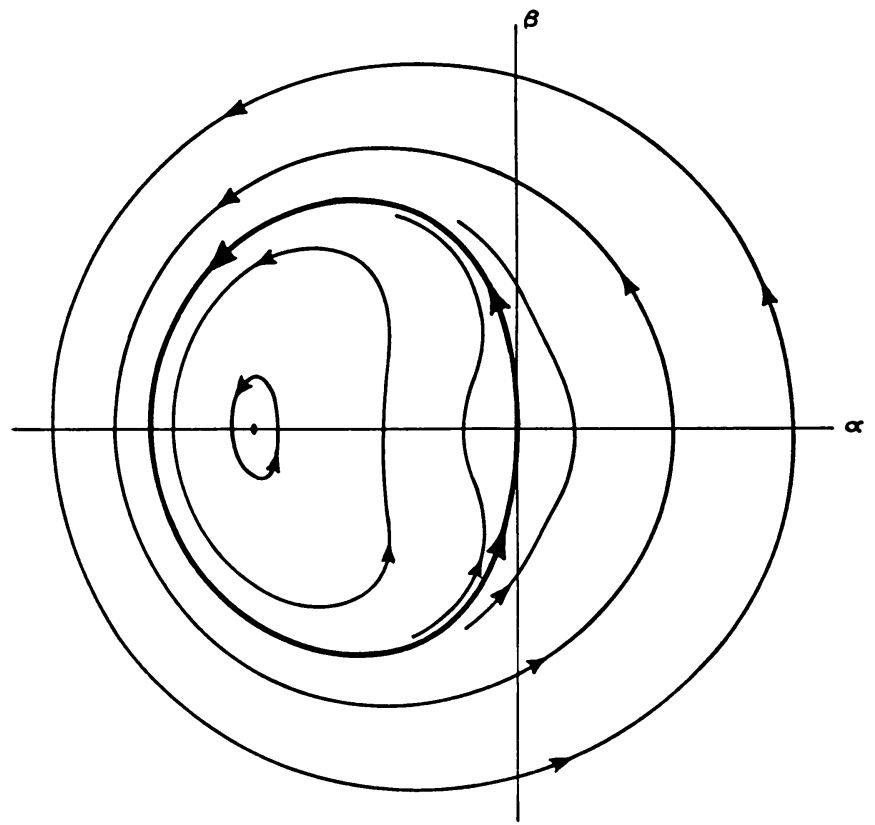

Fig. 4. Trajectories for $m=0$. 
If $m>\eta^{2} / 2$, i.e. $m>\left[1+2 \epsilon-(1+4 \epsilon)^{1 / 2}\right] / \epsilon^{2} \approx 2-4 \epsilon$, then (10) yields two real positive numbers $a$. The larger of these is, as before, a center while the smaller is a saddle point. A periodic (subharmonic) solution of (2) corresponding to the latter is, of course, unstable. In this case, there are three families of closed, phase-plane trajectories. (See Fig. 6). There are the usual large ones which become circular as $c \rightarrow \infty$, the small oval shaped ones which encircle the origin, and the intermediate, rather deformed ones which eventually close down upon the nonzero center. The origin is also a center. The three families of closed trajectories are distinguished by separatrices stemming to and from the saddle point.

As indicated above, the centers and the saddle points of the phase-plane, which are given by (10), are the bifurcation amplitudes corresponding to stable and unstable periodic (subharmonic) solutions of (2). In addition, the origin, which corresponds to the rest position of the pendulum, is stable if $|m|>\eta^{2} / 2$. More generally, nontrivial phaseplane trajectories depict long-period variations in the amplitude $a$ and phase $\gamma$ (or $\Phi$ ) of nonstationary oscillations (3). The closed trajectories depict periodic variations of the amplitude $a$ (beats) while the separatrices and the extraordinary trajectories depict transient (nonperiodic) variations in $a$. In general, the long-period variations of the phase $\gamma$ are similar except that $\gamma$ exhibits a secular (time like) variation along each trajectory which persists in encircling the origin. If a small amount of damping is introduced into the system, the stable centers of the phase-plane become asymptotically stable foci while the characteristics of the unstable singularities remain essentially as

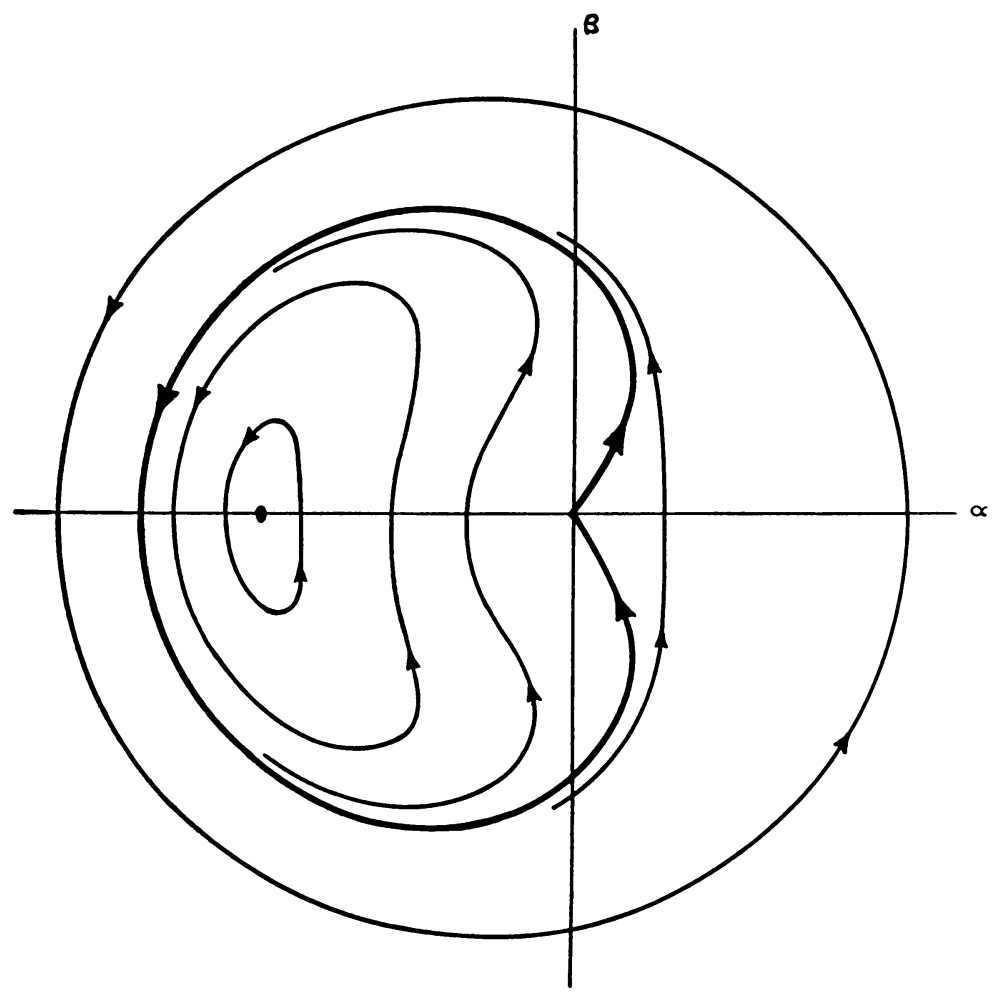

Fia. 5. Trajectories for $0<m<\eta^{2} / 2$. 


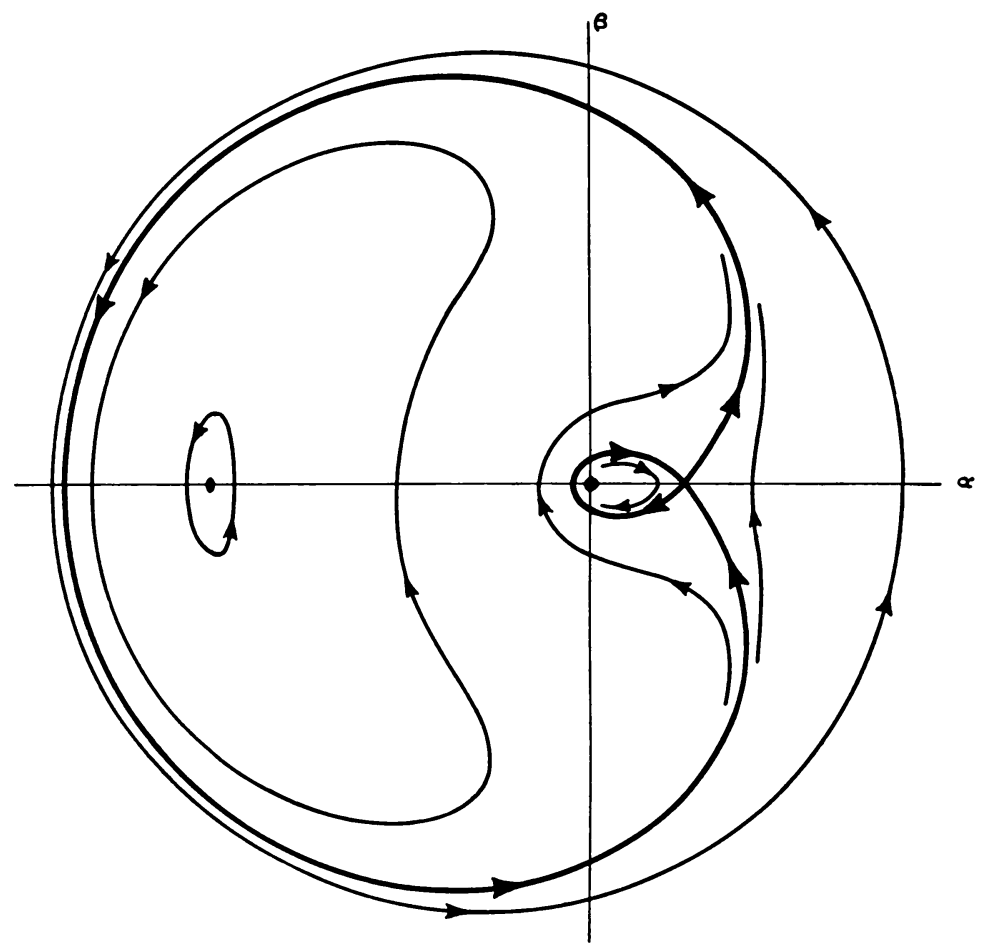

FIG. 6. Trajectories for $\eta^{2} / 2<m$.

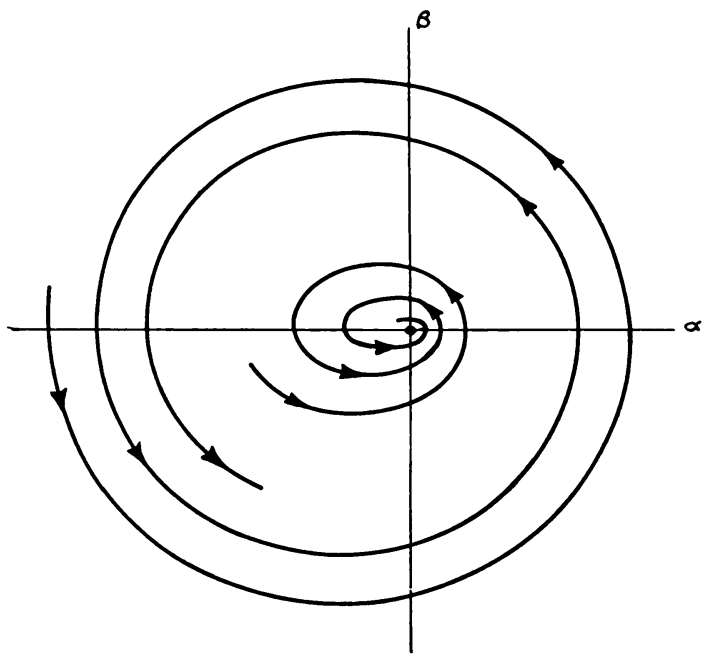

FIG. 7. Trajectories for $m<-\eta^{2} / 2$, with damping. 


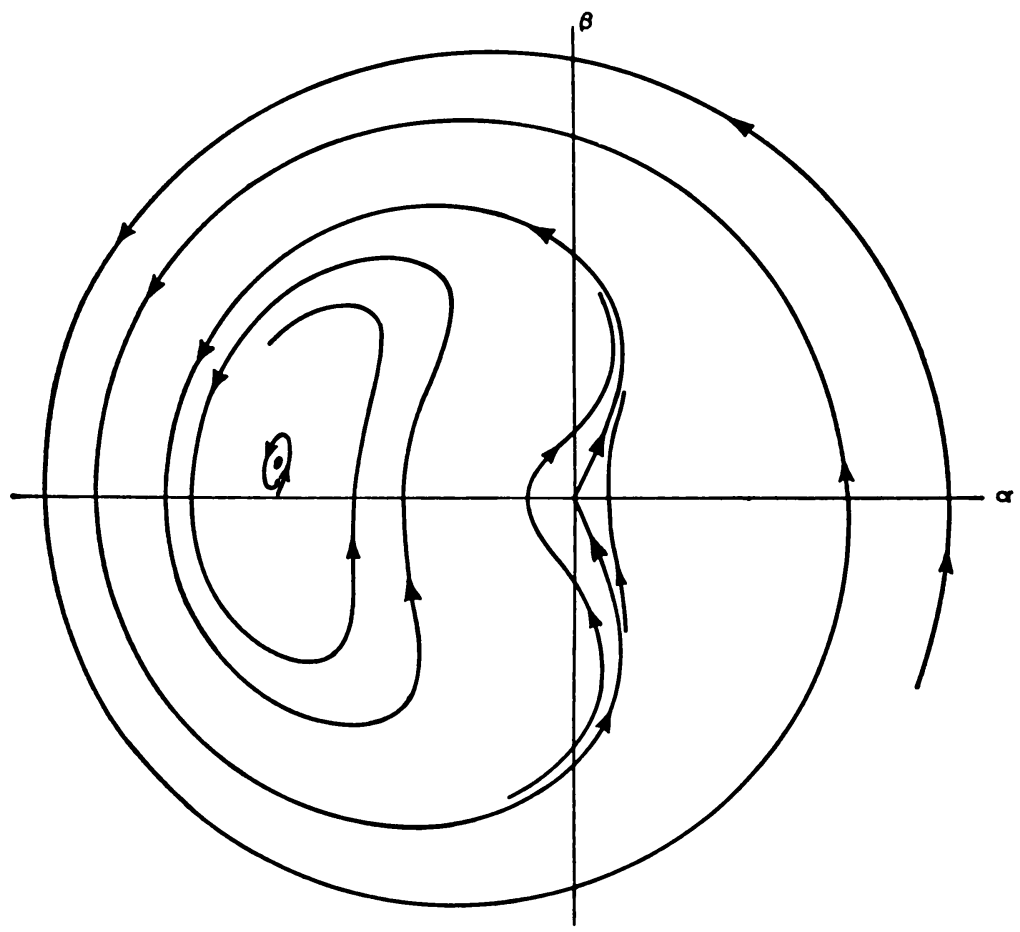

FIG. 8. Trajectories for $0<m<\eta^{2} / 2$, with damping.

before. (See Figs. 7-9) The subharmonic solutions corresponding to the foci are then asymptotically stable and depict the steady-state responses. It is interesting to note that for $|m|>\eta^{2} / 2$, the equilibrium (rest) position is a possible steady-state response also, as in Figs. 7 and 9. In the latter Figure, there are two steady states illustrated.

We complete the integration of the variational system (9) using (11) to eliminate $\gamma$ from the second equation in (9). The variables then separate and we obtain the elliptic integral

$$
\epsilon \tau= \pm \int \frac{d a^{2}}{\left[\frac{\eta^{4}}{4} a^{4}-\left(c+m a^{2}-\frac{a^{4}}{16}\right)^{2}\right]^{1 / 2}}
$$

Thus the square of the amplitude is an elliptic function of $\tau$. Once it has been obtained, $\gamma$ is given directly by (11). However, it is probably sufficient here to obtain expressions for the periods of the long-period variations. For this, (12) is written in the form

$$
\epsilon \tau= \pm \int \frac{16 d a^{2}}{\left\{\left(a^{2}-a_{m}^{2}\right)\left(a_{M}^{2}-a^{2}\right)\left[\left(a^{2}+B\right)^{2}+C\right]\right\}^{1 / 2}}
$$

where $a_{m}$ and $a_{M}$ denote the minimum and maximum respectively of the amplitude for a particular closed trajectory (11). According to the second equation in (9), these are the intercepts of the trajectory with the $\alpha$-axis. The quantities $B$ and $C$ may be obtained in a variety of forms by comparing the coefficients of the polynomial in $a^{2}$ under the radical 
in (13) with those of the corresponding polynomial in (12).* If the constant terms and $a^{6}$ terms are compared, one obtains the forms

$$
B=\frac{a_{m}^{2}+a_{M}^{2}}{2}-16 m, \quad C=\frac{(16)^{2} c^{2}}{a_{M}^{2} a_{m}^{2}}-\left[\frac{a_{m}^{2}+a_{M}^{2}}{2}-16 m\right]^{2} .
$$

Now during one single period the amplitude $a$ varies from $a_{m}$ to $a_{M}$ and again back to $a_{m}$. Thus the period $\Delta \tau$ of the long-period periodic variation in $a$ is given by the equation

$$
\epsilon \Delta \tau=32 \int_{a_{\dot{\mathbf{u}}}}^{a_{\dot{\Delta}}} \frac{d a^{2}}{\left\{\left(a^{2}-a_{m}^{2}\right)\left(a_{M}^{2}-a^{2}\right)\left[\left(a^{2}+B\right)^{2}+C\right]\right\}^{1 / 2} .}
$$

Reduced to standard form [6], this becomes $\epsilon \Delta \tau=64 K(k) / l$, where

$$
K(k)=\int_{0}^{\pi / 2} \frac{d \sigma}{\left(1-k^{2} \sin ^{2} \sigma\right)^{1 / 2}}
$$

is the Legendre complete elliptic integral of the first kind. When exactly one phase-plane trajectory is associated with the particular value of the integration constant $c$, such as the

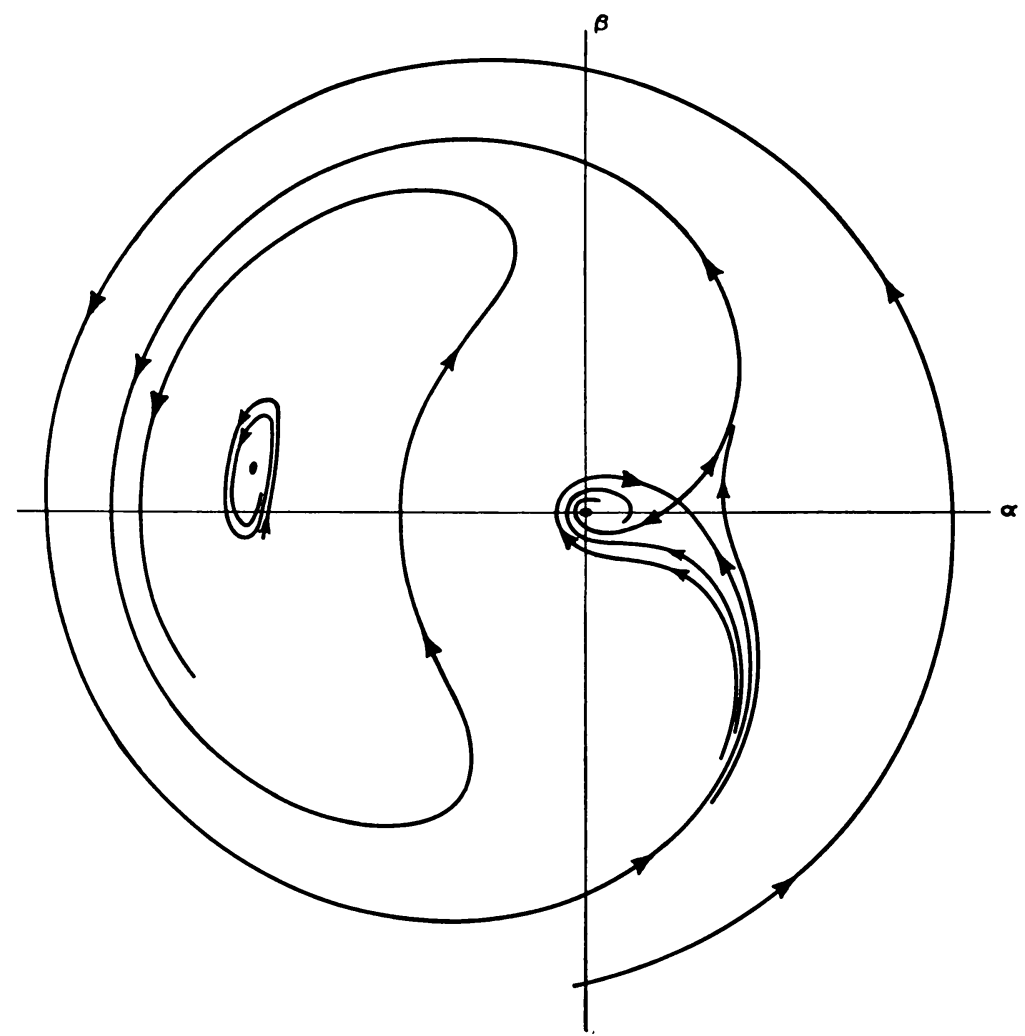

FIG. 9. Trajectories for $\eta^{2} / 2<m$, with damping.

*Note that the leading coefficient (the coefficient of $a^{8}$ ) in (12) is $-1 /(16)^{2}$ and this accounts for the factor " 16 " appearing in (13). 
cases illustrated in Figs. 2-5, then the divisor $l$ and modulus $k$ are given by the expressions

$$
l^{2}=x y, k^{2}=\frac{\left(a_{M}^{2}-a_{m}^{2}\right)^{2}-(x-y)^{2}}{4 x y},
$$

where

$$
x^{2}=\left(a_{M}^{2}+B\right)^{2}+C, \quad y^{2}=\left(a_{m}^{2}+B\right)^{2}+C .
$$

When two phase-plane trajectories are associated with the particular value of the integration constant $c$, such as certain cases illustrated in Fig. 6, then the divisor $l$ and modulus $k$ are given by the expressions

$$
l^{2}=\left(\bar{a}_{M}^{2}-a_{M}^{2}\right)\left(\bar{a}_{m}^{2}-a_{m}^{2}\right), \quad k^{2}=\frac{\left(\bar{a}_{M}^{2}-\bar{a}_{m}^{2}\right)\left(a_{M}^{2}-a_{m}^{2}\right)}{\left(\bar{a}_{M}^{2}-a_{M}^{2}\right)\left(\bar{a}_{m}^{2}-a_{m}^{2}\right)} .
$$

Here we denote by $\bar{a}_{m}$ and $\bar{a}_{M}$ the minimum and maximum of $a$ for the larger trajectory and by $a_{m}$ and $a_{M}$ the corresponding quantities for the smaller trajectory. The periods for the two trajectories are equal though the motions are quite different.

The first order solution is completed by the additive perturbation $X_{1}$. Since the derivatives of $a$ and $\Phi$ are of first order in $\epsilon$, the particular integral

$$
X_{1}=-\frac{a^{3}}{192} \cos 3(\tau-\Phi)-\frac{\eta a}{2(2+\eta)} \cos [(1+\eta) \tau-\Phi]
$$

will satisfy (7) to zero order in $\epsilon$, as required. In any event, $X_{1}$ merely depicts two of the higher harmonics of the response whether the primary oscillations are stationary or nonstationary and is not particularly important to an overall understanding of the motion. It is the principal term $a \cos (\tau-\Phi)=a \cos \frac{1}{2}(\omega t+\gamma)$ which depicts the essential nature of the motion.

4. Comparison with experiments. The results of some interesting experimental studies have been reported in [1]. These are bifurcation amplitudes $A$ for the variable $\theta$ obtained from a physical pendulum mounted on a vibrating table. The corresponding theoretical values are given here by the bifurcation equation (10), with $A=\epsilon^{1 / 2} a$, and reproduce the experimental values remarkably well as indicated in Fig. 10. In fact, the agreement here between theory and experiment is somewhat superior to that reported in [1]. This is because the inevitable truncation of the power series for $\sin \theta$ in (1) is a more serious limitation on the analysis in [1] then the corresponding truncation here in (2) which is a natural consequence of the order of the asymptotic expansion considered. The experimental results suggest that the first order solution obtained here is, perhaps, adequate for most practical purposes.

5. Higher order solutions. Higher order solutions may be obtained by continuing the iteration procedure. For example, having identified $X_{1}$, (in terms of $a, \Phi$ and $\tau$ ) one may now retain all terms in (4) through second order in $\epsilon$. Any near first harmonic, such as in (5), is expressed in terms of the first harmonics with slowly varying coefficients, and the new variational equations for $a$ and $\Phi$ through $\epsilon^{2}$ are obtained from the resulting $\sin (\tau-\Phi)$ and $\cos (\tau-\Phi)$ terms. The remaining (second order) nonresonant terms are incorporated into a perturbational equation for $X_{2}$. The variational equations will be as in (6) except for additional second order terms which will appear on the right. On the left, the higher derivatives $a^{\prime \prime}$ and $\Phi^{\prime \prime}$ and the product terms $a^{\prime} \Phi^{\prime}$ and $\left(\Phi^{\prime}\right)^{2}$ can be evaluated explicitly, to second order, in terms of $a$, $\Phi$ and $\tau$ using the first order 


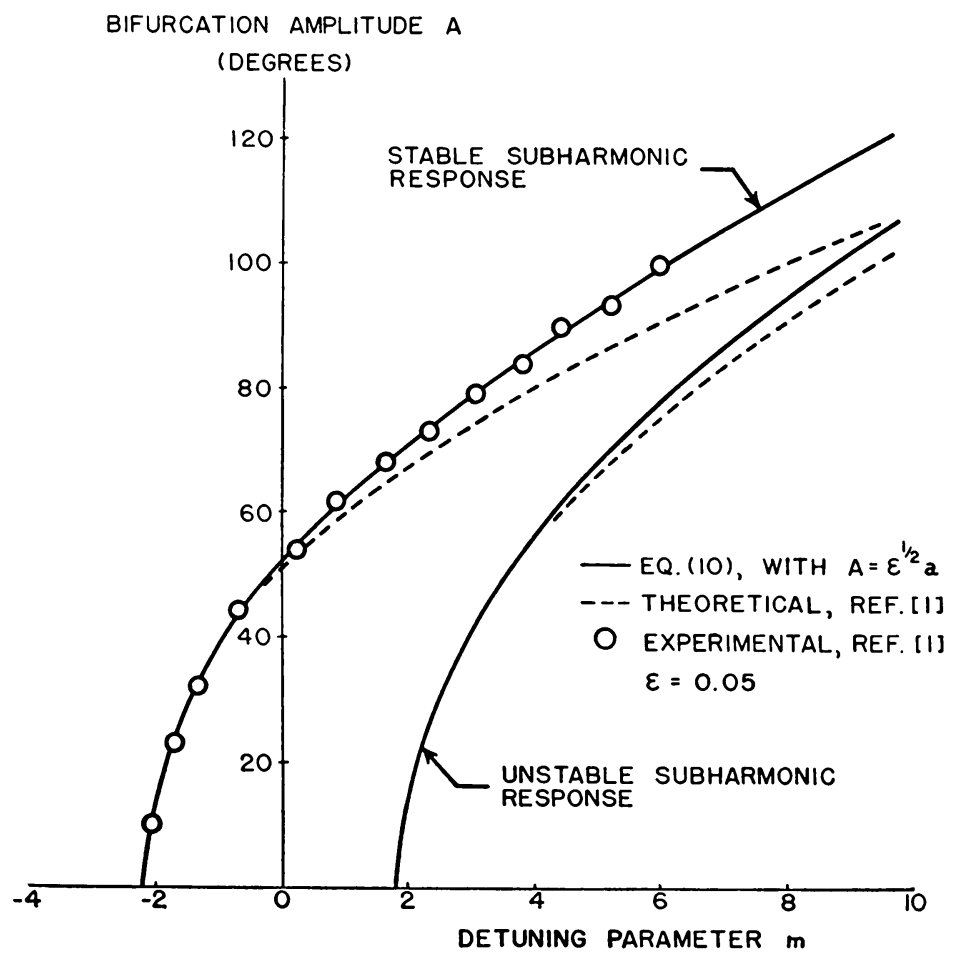

FIg. 10. Bifurcation amplitude.

equations (8). Thus, the second order variational system will reduce to (8) except for additional explicit second order terms on the right. In order to continue the iteration process, only the perturbational equation actually need be integrated and this is always straight-forward. On the other hand, the variational system evidently becomes more involved at each step and integration in closed form of the higher order cases may be extremely difficult. Fortunately, the important qualitative characteristics of the motion are generally reflected in the low order systems.

\section{REFERENCES}

1. R. Skalak and M. I. Yarymovych, Subharmonic oscillations of a pendulum, J. Appl. Mech. 27, 159-164, (1960)

2. R. A. Struble and J. E. Fletcher, General perturbational solution of the harmonically forced van der Pol equation, J. Math. Phys., 2, 880-891 (1961)

3. R. A. Struble and S. M. Yionoulis, General perturbational solution of the harmonically forced Duffing equation, Arch. Rat. Mech. Anal. 9, 422-438 (1962)

4. R. A. Struble: Nonlinear differential equations, McGraw-Hill Book Co. (1962), Chapter 8.

5. N. Bogoliubov and I. Mitropolsky, Asymptotic methods in the theory of nonlinear oscillations, Gos. Izd. Fiz. Mat., Moscow, 1955

6. P. F. Byrd and M. D. Friedman, Handbook of elliptic integrals for physicists and engineers, SpringerVerlag, Berlin, 1954. 